\title{
Photophobia and neuropatic pain in Sudeck's syndrome
}

\author{
de Lossada $\mathrm{CR}^{1 *}$, de Mora MRC ${ }^{1}$, Anil RR ${ }^{1}$, Borroni $\mathrm{D}^{2,3}$, Ayala $\mathrm{CH}^{1}$ and Antonio TD \\ ${ }^{1}$ Hospital Regional Universitario de Málaga, Servicio de Oftalmología, Spain \\ ${ }^{2}$ Royal Liverpool Univsersity Hospital, Department of Doctoral Studies, United Kingdom \\ ${ }^{3}$ Riga Stradins University, Latvia \\ ${ }^{4}$ Hospital Universitario Virgen de la Victoria, Málaga, Spain
}

\begin{abstract}
We present a 14-year-old patient diagnosed with Sudeck syndrome secondary to uneventful foot trauma with complains of decreased visual acuity along with photophobia and intense ocular pain not correlated with the exploratory findings. Sudeck syndrome is an idiopathic neuropathic inflammatory disease characterized by disproportionate pain, unrelated to a previous traumatic event, that can evolve to severe and generalized pain. Recently, a new explanation for those patients with severe eye pain that do not correlate with clinical signs has been described, as "neuropathic eye pain". In the case we present, the pain became widespread and generated photophobia and very intense ocular neuropathic pain, which we believe may be the cause of the visual decrease that our patient presented. We propose that the Sudeck syndrome should become part of the differential diagnosis of neuropathic eye pain.
\end{abstract}

\section{Introduction}

Sudeck syndrome [1-3] is a neuropathic/inflammatory pain disorder characterized by [1-3]: severe pain that extends further than the injured area and disproportionate to the initial event; autonomic dysfunction with sympathetic hyperactivity; neuropathic oedema and motor disorders; atrophy and/or dystrophy. It is a disease of unknown origin, being traumatic injuries, even when banal, the most common cause $(50 \%)$. The cause is unknown in $25 \%$ of the cases [13]. The magnitude of the injury does not seem to be related with the development of the disease. Sympathetic nervous system remains hyperactive after the injury due to unknown reasons [1,3]. Initially, symptoms are confined to the area of the injury although they may generalize if not treated properly [1,2], triggering diffuse and varied symptoms. There is no specific diagnosis, hence being a diagnosis of exclusion. Prompt diagnosis is crucial in order to avoid further complications.

We present a case of a patient presenting intense and disabling photophobia, and neuropathic ocular pain, that was ultimately diagnosed with Sudeck syndrome

\section{Case report}

A 14-year-old patient was diagnosed with Sudeck syndrome by the Paediatric Rheumatologists following a foot trauma three years after the injury, being a diagnosis of exclusion as no other cause was relevant. The injury was treated, yet the foot pain persisted, and would intensify and extend with minor stimuli. Two years after the injury, the patient noticed a visual acuity impairment associated with intense photophobia and was subsequently remitted to our department. The patient was followed-up by Paediatric Rheumatology, Rehabilitation, Pain Management and Paediatric Psychiatry.

In the ophthalmologic exploration, the patient showed a Best Corrected Visual Acuity (BCVA) of 0.6 (Snellen) and +0.50 refraction in both eyes. Other tests were normal: maximum stereopsis in the TNO test, normal pupilar reflex and conserved extraocular movements. Near and far cover test was normal. Slit lamp exploration of the anterior segment and fundoscopy were normal, although the patient was difficult to explore due to intense photophobia and pain. Schirmer and BUT tests were normal. Pain did not subside after applying topical anaesthesia.

Visual field campimetry, Spectral Domain Optic Coherence Tomography (SD-OCT) of the nerve fibre layer and ganglion cell layer, Visual Evoked Potential (VEP), Electroretinography (ERG) and Magnetic Resonance Imaging (MRI) were normal. Neurologists ruled out any pathological findings that could justify the impaired BCVA or photophobia, as trigeminal neuralgia or cluster headache.

During the following medical check-ups, the patient complained of increasing photophobia and pain in both eyes to the extent of obliging the patient to keep sun glasses on even in closed rooms. Ocular exploration was normal in all the check-ups apart from photophobia and ocular pain that wouldn't improve with topical anaesthesia.

\section{Discussion}

Neuropathic pain is defined as "pain triggered or caused by an injury or primary dysfunction of the nervous system" [4]. It may occur in the cornea as it is the most innervated structure of the body [4], and due to damage of other ocular structures. The disease causes pain, ocular irritation, dry eyes and photophobia [5-10].

Sudeck syndrome is an idiopathic neuropathic disorder [1-3] resulting from a hyperactive and disproportionate sympathetic response

*Correspondence to: de Lossada CR, Hospital Regional Universitario de Málaga, Servicio de Oftalmología, Spain, E-mail: carlosrochadelossada5@gmail.com

Key words: sudeck syndrome, neuropathic pain, photophobia, disproportionate pain

Received: February 08, 2019; Accepted: February 15, 2019; Published: February 18,2019 
triggered by an injury, usually trauma. In our case the response was triggered after a food trauma. This response leads to exaggerated pain that can generalized and affect other bodily structures. In our case, pain was referred inducing intense photophobia and neuropathic ocular pain. We even assume that this pain could even be the reason for diminished BCVA. cause

Photophobia is described as abnormal intolerance to light $[5,6,7]$ and it can be accompanied by several ophthalmological diseases such as dry eye, conjunctivitis, uveitis, episcleritis, certain ocular drugs, cataract or refractive surgery. Patients are sensitive to light in situations that other people are not, their ocular exploration is normal, they usually seek for poorly lit environments, and they usually come to their doctor's appointment with their sun glasses on, such as our patient [5,7].

Neurosensory anomalies should be suspected when clinical symptoms are disproportionate to the clinical signs in the ocular surface exploration. A subcategory of dry eye disease has recently been proposed, "neuropathic dry eye", when symptoms are disproportionate to clinical signs $[4,6,9,10]$. These symptoms can aggravate in circumstances of anxiety, depression or post-traumatic stress disorder $[4,6,10]$. Psychological and psychiatric disorders that can handicap the patient can occur in advanced stages of Sudeck syndrome [1]. Our patient was followed-up by Paediatric Psychiatry due to anxiety and depression, two diseases that stand in the differential diagnosis of pain. However, as we have mentioned, the root of the pain and its worsening leading to our patient's disability can also be Sudeck syndrome.

Neuropathic ocular pain may be a consequence of both local (dry eye, after cataract or refractive surgery, infective keratitis, etc) or systemic diseases (Sjögren syndrome, blepharospasm, migraine, fibromyalgia) $[5,6,8,10]$. It has been proven that patients with severe neuropathic ocular pain are more likely to be related to functional diseases, higher non-ocular pain profile, and even mental health issues [10].

The ocular nociceptive sensation travels through the first branch of the trigeminal nerve towards the trigeminal nuclear complex. After synapsis, neurons decussate and join the spinothalamic tracts for synapsis in the thalamus. There is an additional refinement of these nociceptive stimuli in subcortical areas before the pain sensation is produced in the primary sensory cortex. The cornea is the most innerved structure of the human body [4], although it is known that many ocular structures also account for complex areas of nerve endings, such as the conjunctiva, sclera, iris, ciliary body, choroid and retina $[4,8]$.

Eye nociceptors can undergo a plasticity process termed "peripheral sensitization", by which proinflammatory mediators are liberated after tissue damage and eye inflammation, provoking structural changes in corneal nerves, and consequently decreasing pain threshold and intensifying the peripheral pain signal. Over time, peripheral sensitization leads to a "central sensitization", originating a disproportionate response of the central nervous system to peripheral pain signals, such as photoallodyinia, as we believe that happens in our case $[4,6,9,10]$. It has been presupposed that central sensitization takes place in other diseases as for example fibromyalgia, irritable bowel syndrome or temporomandibular pain. Therefore, it is understood that patients that suffer from different forms of chronic pain usually have other overlaying pain diseases, and these can be associated with mood and sleep disorders and decreased quality of life [10]. Topical anaesthesia is used to distinguish peripheral from central ocular neuropathic pain. If pain subsides, it is possible that the pain might have a peripheral origin, whereas if pain does not improve, it probably may be due to a central cause. A mixt component can also be the cause, where pain improvement is noticed after anaesthesia though is still persistent $[4,6,9]$. It has been confirmed that patients that do not witness pain improvement with topical anaesthesia are correlated with higher skin sensitivity to pain and higher discordance between sign and symptoms of the ocular surface when compared to patients that experience relief after anaesthesia $[9,10]$.

Treatment of Sudeck syndrome $[1,4,8,9,10]$ is based on rehabilitation of the affected limb, analgesic management and, when necessary, sympathetic blockade and sympathectomy in advanced cases. Considering ocular treatment, managing the underlying cause is imperative. For instance, dry eye syndrome or Meibomian gland dysfunction will require topical treatment with preservative-free artificial tears, steroids, cyclosporine, autologous serum, nerve grow factor, platelet and growth factor rich plasma. In severe patients with involvement of central symptoms, tricyclic antidepressants, anticonvulsants, opioids or alternatives such acupuncture [4] may be used. A multidisciplinary follow-up is recommended for a correct management.

We believe that Sudeck syndrome could be included in the differential diagnosis of neuropathic ocular pain as it is defined as a neuropathic disease consisting of an exaggerated response to pain after an injury, to a degree that it does not match the intensity of the symptoms that the patients experience.

\section{Conclusion}

We propose that Sudeck syndrome could be a cause of photophobia and neuropathic ocular pain and decrease the quality of life of patients, hence it should be included in the differential diagnosis of neuropathic ocular pain.

\section{References}

1. Fornos-Vieitez B, López-Díez FE, Ciriza-Lasheras A, López-López D (2008) Síndrome de Sudeck (distrofia simpatico refleja). Semergen 34: 455-8

2. Oerlemans HM, Oostendorp RA, de Boo T, Goris RJ (1999) Pain and reduced mobility in complex regional pain syndrome I: outcome of a prospective randomised controlled clinical trial of adjuvant physical therapy versus occupational therapy. Pain 83: 77-83. [Crossref]

3. van de Meent H, Oerlemans M, Bruggeman A, Klomp F, van Dongen R, et al. (2011) Safety of "pain exposure" physical therapy in patients with complex regional pain syndrome type 1. Pain 152: 1431-1438. [Crossref]

4. Dieckmann G, Goyal S, Hamrah P (2017) Neuropathic Corneal Pain: Approaches for Management. Ophthalmology 124: S34-34S47. [Crossref]

5. Katz BJ, Digre KB (2016) Diagnosis, pathophysiology, and treatment of photophobia. Surv Ophthalmol 61: 466-477. [Crossref]

6. Morkin MI, Hamrah P (2018) Neuropathic dry eye: When serum defeats tears. Ophthalmol Times.

7. Lebensohn J (1943) The nature of photophobia. Arch ophthalmol 12: 380-3.

8. Assam JH, Bernhisel A, Lin A (2018) Intraoperative and postoperative pain in cataract surgery. Surv Ophthalmol 63: 75-85. [Crossref]

9. Crane AM, Feuer W, Felix ER, Levitt RC, McClellan AL, et al. (2017) Evidence of central sensitisation in those with dry eye symptoms and neuropathic-like ocular pain complaints: incomplete response to topical anaesthesia and generalized heightened sensitivity to evoked pain. Br J Ophthalmol 101: 1238-1243. [Crossref]

10. Crane AM, Levitt RC, Felix ER, Sarantopoulus KD, McClellan AL, et al. (2017) Patient with more severe symptoms of neurophatic ocular pain report more frequent and severe chronic overlapping pain conditions and psychiatric disease. Br J Ophthalmol 101: 227-231. [Crossref]

Copyright: (C)2019 de Lossada CR. This is an open-access article distributed under the terms of the Creative Commons Attribution License, which permits unrestricted use, distribution, and reproduction in any medium, provided the original author and source are credited. 